\title{
USO DE TECNOLOGÍAS 3D EN LA DIGITALIZACIÓN Y DIFUSIÓN DE DOCUMENTOS DE ALTO VALOR PATRIMONIAL
}

\author{
José-Manuel Pereira-Uzal y Jesús Robledano-Arillo
}

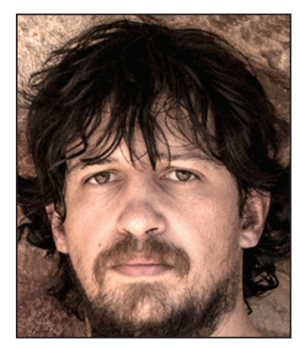

José-Manuel Pereira-Uzal es restaurador de patrimonio cultural, documentalista y fotógrafo. Experto en tecnologías de la imagen y su aplicación al patrimonio cultural, es máster en documentación audiovisual por la Universidad Carlos III de Madrid. Responsable de DigitalHeritage, sitio web especializado en el asesoramiento e investigación sobre la aplicación de tecnologías de la imagen al patrimonio cultural y documental.

http://orcid.org/0000-0002-9240-5691

DigitalHeritage Pedreira Calo, 99. 15866 Teo, A Coruña, España http://www.jpereira.net

info@jpereira.net

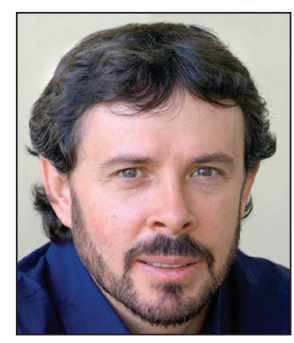

Jesús Robledano-Arillo, doctor en documentación, es profesor del Departamento de Biblioteconomía y Documentación de la Universidad Carlos III de Madrid. Imparte docencia en estudios de grado y de postgrado relacionada con la tecnología de la imagen y la digitalización de documentos. Su trabajo se centra principalmente en la digitalización de patrimonio documental y documentación audiovisual.

http://orcid.org/0000-0002-4090-8684

Univ. Carlos III de Madrid, Depto. de Biblioteconomía y Documentación Av. de la Universidad Carlos III, 22 28270 Colmenarejo, Madrid, España jroble@bib.uc3m.es

\section{Resumen}

Se analizan las limitaciones de las imágenes digitales raster bidimensionales para la representación gráfica de documentos con valor patrimonial que presentan volumen o elementos tridimensionales, proponiéndose un modelo de documentación gráfica 3D que complementa la información visual raster 2D que se ofrece a los usuarios en los servicios de archivos y bibliotecas digitales. Este modelo aplica de forma concurrente técnicas de fotografía estereoscópica, RTI (reflectance transformation imaging) y modelos 3D obtenidos mediante procedimientos de captura SfM (structure for motion).

\section{Palabras clave}

Imagen digital, Digitalización, Patrimonio documental, 3D, Structure from motion, X3D.

\section{Title: Use of 3D technologies in high patrimonial value document digitalization and dissemination}

\section{Abstract}

We analyze the limitations of two-dimensional (2D) raster digital images for graphic representation of documents with volume or three-dimensional (3D) elements, proposing a visual model based on 3D techniques that complements the visual information conveyed by the 2D raster images that are usually offered in digital archives or library services. The proposed model concurrently applies stereoscopic photography techniques, reflectance transformation imaging (RTI) and 3D models obtained by structure for motion (SfM) procedures.

\section{Keywords}

Digital imaging, Digitization, Documentary heritage, 3D, Structure from motion, X3D.

Pereira-Uzal, José-Manuel; Robledano-Arillo, Jesús (2013). “Uso de tecnologías 3D en la digitalización y difusión de contenidos de alto valor patrimonial”. El profesional de la información, mayo-junio, v. 22, n. 3, pp. 215-223. 


\section{Introducción}

La imagen digital se ha convertido en el medio por excelencia para la documentación y difusión del patrimonio documental. La mayoría de las instituciones culturales están digitalizando su patrimonio documental y difundiéndolo a través de servicios de biblioteca o archivo digital en formatos raster, tales como jpeg o tiff. Podemos hablar en la actualidad de un paradigma en la representación digital y difusión de documentos patrimoniales basado en este tipo de formatos. En una imagen raster la representación es bidimensional, a través de una matriz de píxeles (puntos de información de color e intensidad de luz posicionados en el espacio bidimensional mediante dos coordenadas). La visualización de la imagen raster de un documento obtenida fotográficamente es realista, en el sentido de que hay una gran proximidad visual con la vista del documento físico digitalizado. No obstante, este tipo de representaciones adolece de datos volumétricos precisos. Además su visualización en pantalla o su reproducción impresa tendrán distorsiones en las formas de los documentos que no pueden quedar perfectamente planos durante su captura, debido a sus propias características materiales intrínsecas, un estado de conservación deficiente, o a que contienen elementos o superficies con volumen, como sellos tridimensionales, encuadernaciones, dobleces, información en relieve, piezas de cobertura o decorativas en relieve, estuches, marcos o soportes de gran grosor.

Una reproducción 2D aporta información sobre el contenido de un documento, pero la 3D relaciona ese contenido con el espacio

El conocimiento de las características formales y tridimensionales de los documentos es de gran interés para el análisis en profundidad que se puede requerir desde determinadas disciplinas científicas, tales como la codicología, diplomática, paleografía, sigilografía, conservación y restauración, historia del arte, historia de la fotografía, o la historia antigua y medieval. En estos estudios la representación bidimensional en formato raster es insuficiente y poco representativa de la información material y formal que se requiere.

Las imágenes 3D facilitan un análisis espacial en las tres dimensiones del espacio de la realidad física representada, por lo que son un sustituto ideal del propio documento físico para muchos de los estudios que requieren su acceso y manipulación, así como para las funciones de preservación, creación de facsímiles físicos, impresión mediante tecnologías 3D, y restauración física y digital de los documentos originales. Un conservador de documentos, por ejemplo, podría utilizar un modelo 3D preciso para el diagnóstico de alteraciones estructurales y morfológicas de un documento, como el grado de encogimiento o pérdida de sustancia de un soporte, o para el mejor conocimiento del tipo de reparaciones que se le han aplicado; un historiador o codicólogo podría conocer con un alto grado de precisión aspectos tridimensionales del documento que le ayudan a determinar con exactitud las técnicas de factura del documento o de los elementos accesorios que presenta, tales como sellos, cuerdas, guardas de tela, plica plegada, exlibris con relieve, o encuadernaciones con diversas técnicas decorativas en relieve (repujados, herrajes, cierres, incrustaciones de orfebrería o joyas).

También son de utilidad para las tareas de difusión de los documentos, al público general o en el ámbito de la educación y aprendizaje, por su vistosidad y por ofrecer un acercamiento mayor a la materialidad del documento. Mientras que una reproducción 2D aporta información sobre el contenido de un documento, la información 3D relaciona ese contenido con el espacio, presentándose como una solución ideal para mejorar la comprensión del documento como objeto y no como un mero contenedor de información. Un ejemplo de uso de imágenes 3D interactivas para la difusión de una selección de documentos de gran valor patrimonial se tiene en la Biblioteca del Estado de Baviera, sólo que los modelos son relativamente limitados, poco informativos, y de poca utilidad más allá de una finalidad divulgativa y educativa.

http://www.bayerische-landesbibliothek-online.de/3d

La aplicación de estas técnicas supone un coste en términos de tiempo y esfuerzo, por lo que conviene aplicarlas de forma selectiva, priorizando aquellos documentos sobre los que se estime una mayor frecuencia de análisis físico o que tengan un mayor impacto social debido a su valor; así se explotará su potencial en un mayor grado sin generar unos costes excesivos.

La tecnología 3D se utiliza ya para la documentación gráfica y difusión de patrimonio arqueológico, escultórico y arquitectónico desde hace más de dos décadas. Muchos museos la están aplicando para la difusión de obras en sus sitios web. Ya se cuenta con buenos estados de la cuestión sobre ello (Stanco; Battiato; Gallo, 2011; loannides et al.,

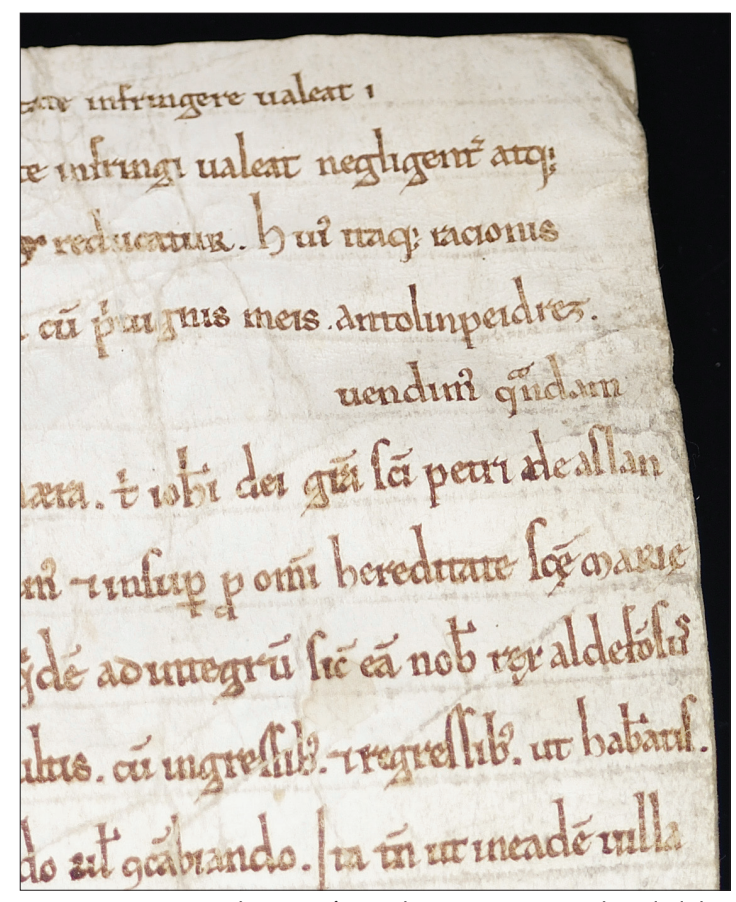

Figura 1. Captura raster de una página de pergamino medieval alabeada 


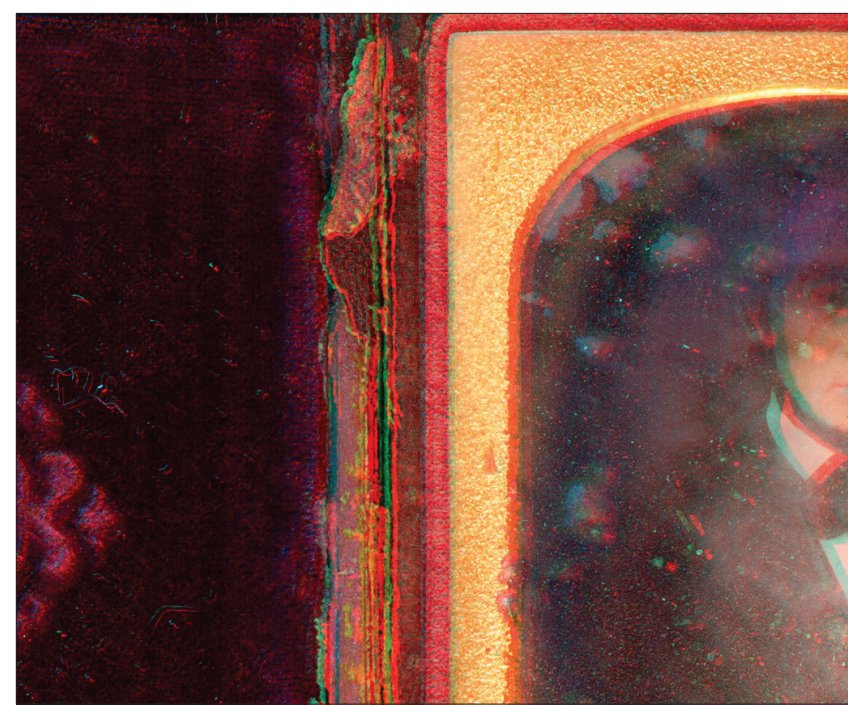

Figura 2. Detalle de anaglifo obtenido de la digitalización de una fotografía estuchada (daguerrotipo)

2012); incluso el proyecto Carare, asociado a Europeana, ha definido un marco tecnológico para la difusión de modelos 3D de este tipo de obras mediante Europeana (Pletinckx, 2011). Su aplicación a la digitalización de documentos está mucho menos generalizada, y ha sido principalmente empleada como un método intermedio de ayuda a los procesos de restauración digital de documentos valiosos muy deteriorados (Bianco et al., 2010; Brown et al., 2007; Pal; Terras; Weyrich, 2013; Tian; Narasimhan, 2011; Zhang; Tan, 2005). En las líneas de trabajo existentes que han aplicado 3D a la digitalización de documentos no hemos detectado una propuesta orientada a la difusión y documentación gráfica precisa de las características materiales de los documentos. Tampoco hemos visto la descripción de métodos de procesamiento que hagan factible la digitalización 3D con tecnologías consolidadas, de bajo coste, alta eficiencia y al alcance de cualquier institución.

El objetivo de este trabajo es definir un modelo de documentación visual y de difusión de información formal de documentos patrimoniales de gran valor basado en tecnologías de imagen 3D. La aplicación de este modelo facilitará la creación de imágenes 3D que, de forma complementaria a la digitalización 2D en formato imagen digital raster, puedan proporcionarse a los usuarios de los fondos para procurarles un acercamiento preciso a las características morfológicas y volumétricas de estos objetos. El modelo que describimos incorpora estándares web para la representación y difusión de información y software libre de fácil acceso.

\section{Limitaciones de la imagen bidimensional de documentos}

La imagen bidimensional no es apta para representar con fidelidad las características tridimensionales ni la forma geométrica que pueden tener muchos tipos de documentos cuando se digitalizan superficies no perfectamente planas. Cuando se capturan objetos tridimensionales mediante un sistema fotográfico y se representan en una imagen 2D se produce una distorsión a nivel espacial, pues la tercera di-

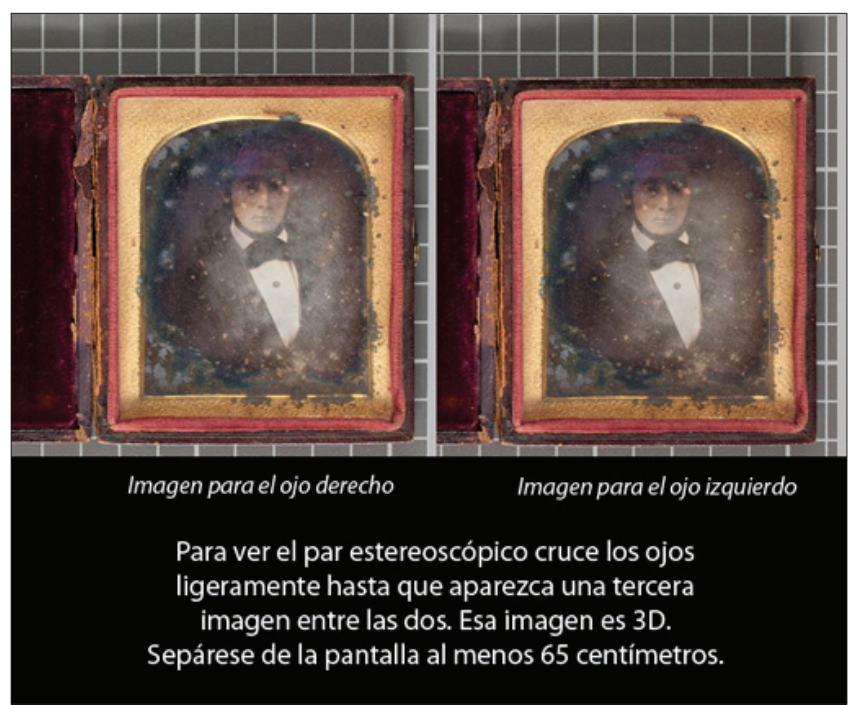

Figura 3. Par estereoscópico obtenido de la misma fotografía de la figura 2, preparado para su visualización 3D mediante la técnica de autoestereoscopia

mensión tiene que proyectarse necesariamente sobre un espacio con una dimensión menos. El tipo de proyección de la escena real captada en la imagen que realiza la óptica de la cámara fotográfica sobre el plano del sensor es de tipo perspectivo o central, lo que implica cierta transformación de las relaciones espaciales entre las partes que no quedan a la misma altura y distancia con respecto al eje óptico del objetivo del dispositivo de captura. En una proyección perspectiva los elementos representados van cambiando de tamaño, forma, punto de vista y posición sobre la imagen bidimensional proyectada en función de su distancia, en la vertical y en la horizontal, al objetivo de la cámara.

Incluso en la representación de una superficie alabeada, como la que se suele tener en la captura de un libro abierto, tendremos estos factores de deformación en acción. Veámoslo con un ejemplo. En la imagen raster de la figura 1, resultado de la captura fotográfica digital de un documento alabeado en soporte pergamino, se puede apreciar la alteración de la geometría de la página rectangular del códice y el cambio de escala, posición y punto de vista de las letras y elementos que no quedan a la misma distancia de la cámara en los ejes horizontal y vertical.

Este tipo de deformaciones geométricas deberá corregirse si la imagen va a ser usada para la realización de análisis espacial bidimensional. La corrección manual puede ser ineficiente y poco precisa, por lo que se requiere el empleo de procedimientos automatizados de ortorrectificación. La ortorrectificación hace uso de información tridimensional del objeto para eliminar las distorsiones horizontales y verticales debidas a su relieve, de manera que la imagen bidimensional presente las mismas propiedades geométricas que un plano cartográfico. Pero incluso una imagen fotográfica ortorrectificada carecerá de información volumétrica que puede ser esencial para el conocimiento de la geometría del objeto. En consecuencia, el uso de tecnologías 3D es un paso inevitable para poder aportar información formal y volumétrica precisa y sin distorsiones de este tipo de documentos. 


\section{Técnicas para generar la ilusión de espacio tridimensional: fotografía estereoscópica y RTI (reflectance transformation imaging)}

Las imágenes obtenidas mediante estas técnicas son representaciones en formato raster bidimensionales, por lo que carecen de información volumétrica calibrada. En consecuencia, no pueden ser consideradas como modelos con información geométrica 3D de alta precisión. No obstante, son de gran utilidad para el análisis espacial tridimensional de las superficies de los documentos, pues al crear sus imágenes en el observador la ilusión de un espacio tridimensional, facilitan la realización de determinadas funciones analíticas en ese espacio sobre la propia imagen bidimensional representada en una pantalla.

Los anaglifos, los pares estereoscópicos y las imágenes RTI pueden ser obtenidos desde imágenes digitales capturadas a muy alta resolución

La fotografía estereoscópica aplica el mismo principio de la visión estereoscópica humana para generar imágenes tridimensionales: se obtienen dos imágenes de la misma escena con un desplazamiento de, aproximadamente, seis centímetros y medio, de forma que cada una de ellas capte la perspectiva correspondiente a cada ojo. Durante la visualización un mecanismo aporta la ilusión óptica de las tres dimensiones al proporcionar a cada ojo exclusivamente su imagen correspondiente. Es relativamente fácil digitalizar y difundir imágenes estereoscópicas de los documentos mediante la técnica de los anaglifos y de los pares estereoscópicos. Disponemos ya de una amplia bibliografía sobre estereoscopía, con trabajos apropiados para diferentes niveles de conocimiento sobre la materia (Alkhadour et al., 2009; StereoGraphics Corporation, 1997; Edirisinghe; Jiang, 2000; Farnsworth).

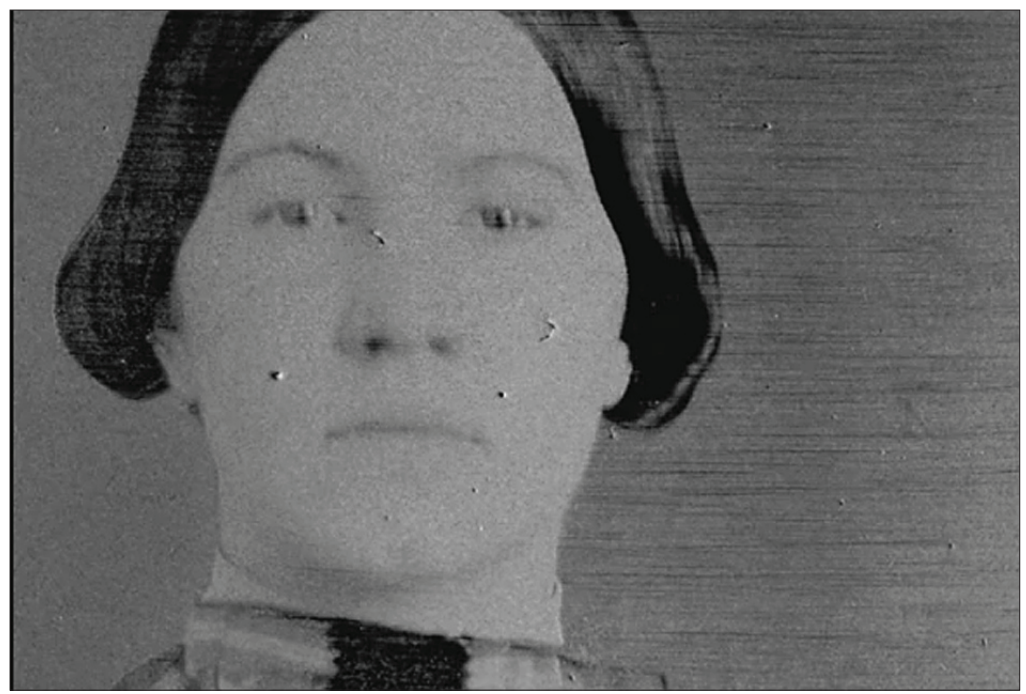

Figura 4. Visualización de imagen RTI de un daguerrotipo²
Los anaglifos superponen las dos imágenes estereoscópicas sobre la misma superficie con una ligera diferencia de registro, teñidas respectivamente de rojo y cian. La sensación de tridimensionalidad se produce cuando se visualizan con unas gafas con filtros de estos mismos colores. Gracias a los fenómenos de síntesis aditiva de nuestra visión, se neutralizan parcialmente los colores de los filtros percibiéndose una imagen tridimensional con un color cercano al original. Desafortunadamente, la capacidad de nuestra visión por adaptarse a ciertas dominantes hace que el sistema visual no pueda eliminar del todo la percepción de los colorantes, impidiendo un registro exacto del color.

Los pares estereoscópicos contienen las dos imágenes captadas una junto a otra y sin separación; a través de un visor estereoscópico o de un procedimiento de visión cruzada a ojo desnudo - que hacen que cada ojo vea exclusivamente su imagen correspondiente- se genera la sensación de tridimensionalidad. Al no precisarse de transformación de color, se pueden obtener colores muy próximos a los de los objetos. Su limitación es la escasa resolución espacial que puede aportarse en una visualización completa del documento, lo que restará cualquier utilidad para su análisis espacial, ya que su visualización en pantalla requiere que las imágenes del par sean visualizadas a muy pequeño tamaño. Por ello, sólo podemos considerar este sistema para un uso divulgativo, salvo que se realicen diferentes vistas estereoscópicas de detalles pequeños del documento a alta resolución.

La técnica RTI fue propuesta inicialmente en 2001 por Tom Malzbender de Hewlett Packard Labs para realzar texturas o relieves mediante la proyección controlada de sombras (Cultural heritage imaging, 2012). Se tienen múltiples ejemplos de su aplicación con éxito para la documentación visual de patrimonio arqueológico y documental (Piquette; Crowther, 2011; Earl et al.; Mudge et al., 2006). Los modelos RTI utilizan el concepto de iluminación interactiva, a base de múltiples tomas de un objeto iluminado por una fuente de luz desde distintos ángulos e inclinaciones. Durante su visualización se puede interaccionar con la imagen para evidenciar diferentes aspectos de la superficie no visibles en condiciones normales de iluminación.

Los anaglifos, los pares estereoscópicos y las imágenes RTI pueden ser obtenidos desde imágenes digitales capturadas a muy alta resolución; por ello resultan de gran utilidad para la realización de determinadas funciones analíticas - una vez corregidas las distorsiones geométricas que puedan presentar-, especialmente en la identificación de tipos de tratamientos, texturas y deterioros. Por ejemplo, si observamos la imagen del anaglifo del daguerrotipo en color (figura 2) con unas gafas de filtros de colores percibiremos con facilidad cómo se disponen las distintas capas de material traslúcido o semitraslúcido en el espacio, pudiendo identificar sin problemas a qué capa corresponden los deterioros visibles que presenta el documento original. Este análisis es imposible hacerlo sobre la imagen raster no estereoscópica. 


\section{Técnicas para la obtención automática de modelos 3D precisos}

\section{Técnicas basadas en tomas fotográficas}

La estereofotogrametría es la disciplina encargada de calcular las dimensiones y posiciones de los objetos en el espacio a partir de medidas realizadas sobre tomas fotográficas estereoscópicas. Existen en este momento en el mercado numerosas aplicaciones de modelado 3D automatizado que hacen uso de técnicas estereofotogramétricas, como Photomodeler de la empresa Eos Systems, Photoscan de Agisoft o 123Catch de Autodesk.

http://www.photomodeler.com

http://www.agisoft.ru/products/photoscan

http://www.123dapp.com/catch

Sin embargo en las últimas décadas los avances en visión artificial han lanzado al mercado nuevas propuestas de modelado 3D, como Structure from motion (SfM) (Wu, 2011; Wu et al., 2011). Esta técnica se basa en la manera en que los sistemas de visión humanos o animales reconstruyen estructuras tridimensionales a partir de imágenes 2D proyectadas en la retina, gracias al movimiento de estas estructuras respecto al observador, o de éste respecto a aquellas. Aunque ya existen trabajos entorno al SfM en la década de los ochenta, es ahora cuando comienzan a aparecer programas consistentes capaces de resolver modelos 3D complejos con una cierta eficacia y agilidad. Una de las principales aportaciones ha sido la de Changchang Wu con el Multicore bundle adjustment (Wu et al., 2011) y el software libre VisualSFM ${ }^{3}$.

\section{Escaners y cámaras 3D}

Aunque en lo últimos años las estrategias lanzadas al mercado del hardware 3D han sido múltiples y dispares, poco a poco el abanico de posibilidades se va reduciendo a dispositivos basados en haces de laser pulsado, conocidos por el acrónimo de Lidar (Light detection and ranging) o escaners láser 3D. Estos dispositivos registran las coordenadas tridimensionales de una muestra de puntos mediante el cálculo de la distancia entre superficie y emisor a través de la estimación del tiempo de retraso entre la emisión y recepción del haz laser. Una alternativa

a los dispositivos Lidar son los escaners de luz estructurada, que analizan las deformaciones de un patrón de luz proyectada sobre una superficie. A través de este sistema se pueden generar modelos 3D de objetos cercanos con cierta eficacia.

Ambas tecnologías proporcionan resultados excelentes, pero son de coste alto y de uso complejo. Por ello, las estrategias de modelado 3D sobre imágenes 2D realizadas con equipos fo-

Figura 5. Detección de características SIFT tográficos convencionales son actualmente una alternativa razonable para la generación de modelos 3D de bienes patrimoniales.

\section{Modelos 3D de nubes de puntos y mallas}

Una nube de puntos no es más que una forma coloquial de denominar a una colección de cientos, miles, o millones de coordenadas espaciales tipo XYZ. La precisión de los modelos 3D depende del número de puntos usado para describirlos. Adicionalmente estos modelos pueden estar acompañados por la información de color de cada punto representada en el sistema RGB (red, green, blue), lo que permite además disponer de la información colorimétrica de la porción de la superficie a la que representa dicho punto.

Un modelo en malla es una reconstrucción de una superficie a partir de la información de una nube de puntos. Una malla está formada por un conjunto de caras que convergen en un vértice, que corresponde a uno de los puntos de la nube.

Las reconstrucciones de superficies se realizan con algoritmos particulares como el conocido Poisson (Kazhdan; Bolitho; Hoppe, 2006), disponible en la aplicación de software libre Meshlab. La representatividad de la reconstrucción dependerá de la densidad de la nube y de la complejidad del modelo. Las estimaciones geométricas, volumétricas o estructurales precisas entre dos modelos se realizan a partir de las nubes de puntos con herramientas como CloudCompare $^{4}$, ya que éstas aportan la precisión y fidelidad necesarias para tal operación. Las mallas son un medio apropiado para la virtualización de un objeto sobre la que podemos aplicar texturas fotorrealísticas, bañarlas con fuentes de luz que darán lugar a la proyección de sombras, etc. http://meshlab.sourceforge.net

\section{Generación de un modelo 3D de un documento por técnicas de SfM}

Cualquier proyecto de fotogrametría estereoscópica comienza con la toma de imágenes del objeto a representar. La técnica SfM permite una gran flexibilidad en la sesión de captura fotográfica, sin embargo, se deben cuidar los movimientos de paralaje de la cámara así como la convergencia entre las tomas, con el fin de asegurar un solapamiento

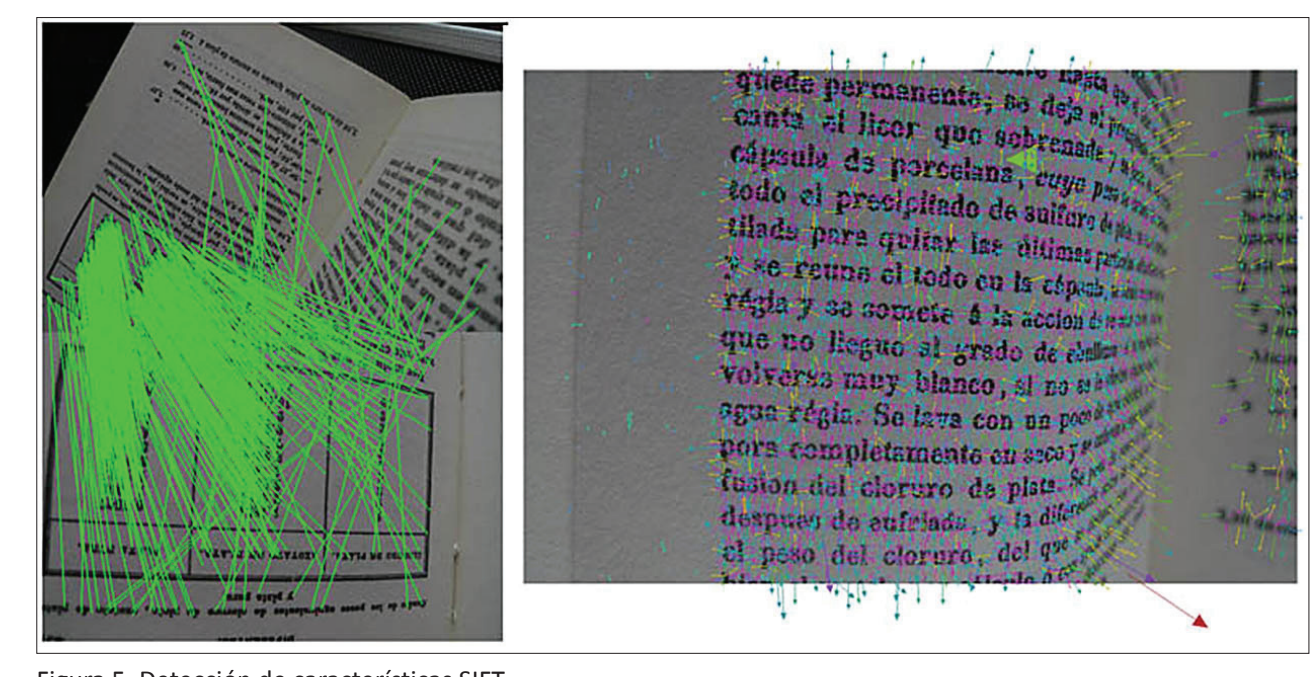




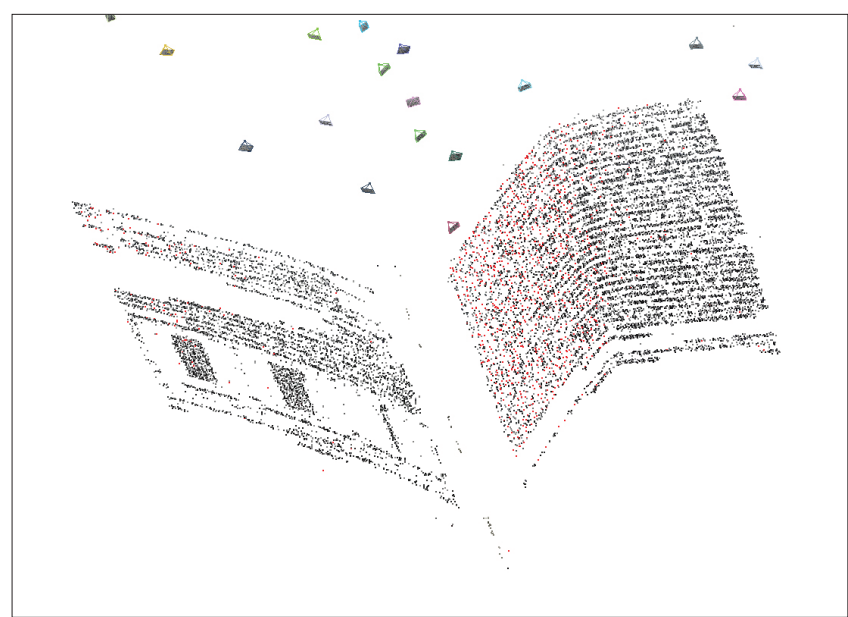

Figura 6. Ajuste Bundler

suficientemente coherente. Los problemas de exposición condicionan el número de características comunes entre las imágenes, y pueden provocar inconsistencias en las texturas, por lo que, al igual que la gestión del color, la exposición debe ser minuciosamente controlada. Aunque en el mercado existen programas, como Agisoft Photoscan o Autodesk 123D Catch, que poseen una clara inspiración en las rutinas de SfM, es recomendable que la iniciación a estas técnicas de modelado 3D se haga con aplicaciones libres, como $\mathrm{Vi}$ sualSFM, que facilitan la aproximación y comprensión de dichos procesos sin coste de licencia.

El flujo de trabajo de una aplicación basada en SfM comienza por la detección automática de características gráficas comunes entre las imágenes de entrada, denominado SIFT (scale-invariant feature transform).

El segundo paso es lo que se conoce como ajuste bundler (empaquetar, juntar), un concepto íntimamente ligado a la fotogrametría, consistente en la recolocación espacial tanto de los puntos coincidentes entre imágenes como de las posiciones de las cámaras que tomaron dichas imágenes, proyecciones, etc. Esta información se utiliza también en la creación automática de ortoimágenes o texturizados a partir de las imágenes raster de entrada. El resultado de dicho proceso es una nube de puntos discreta, o poco densa, cuya densidad dependerá del número de puntos clave extraídos por el proceso anterior.

Estas nubes discretas puedan arrojar información sobre la geometría del objeto representado, pero son insuficientes para poderlo analizar en detalle. Para realizar esta función se requiere, a continuación, crear una nube de puntos densa que, al contener millones de puntos, tiene una mayor capacidad descriptiva de superficies y geometría, aportando además la colorimetría precisa de su superficie si se hizo una correcta gestión del color en la toma y manipulación de las fotografías empleadas en el proceso.

Inicialmente, el modelo recién creado, aunque preciso en proporciones, no posee una escala ni coordenadas conocidas, por lo que se hace necesario atribuirle una escala o contextualizarlo en un sistema de coordenadas, con el fin de poder realizar mediciones sobre el modelo, o realizarlo con otros modelos de un mismo escenario.

Opcionalmente, se puede continuar el flujo de trabajo mediante la virtualización del objeto, consistente en la regeneración de su superficie con una malla.

Gracias a la información generada durante el ajuste bundler se dispone de contenido suficiente para regenerar la textura del modelo recién creado partiendo de las posiciones de las cámaras y de las imágenes digitales raster de entrada. A partir de esta información se puede también realizar un proceso de ortorrectificación automática, del que se obtendrá una única imagen fotográfica digital corregida en distorsiones geométricas y perspectivas.

\section{Propuesta sobre un sistema de documentación 3D del patrimonio documental}

De acuerdo con las estrategias abordadas a lo largo de este artículo, proponemos un modelo de uso de las tecnologías 3D en el ámbito de la documentación gráfica de documentos con volumen de alto valor patrimonial basado en los siguientes componentes:

a) Componentes para un análisis técnico de precisión.

- Un modelo 3D basado en nubes de puntos densas, elaborado con una serie de imágenes raster sobre las que se ha hecho una cuidadosa gestión del color. Este modelo aporta una vista geométrica en volumen e información colorimétrica precisa del exterior del documento en todas las caras del modelo.

- Ortofotografías de las vistas más relevantes del documento. Permite realizar un análisis espacial preciso de las su-
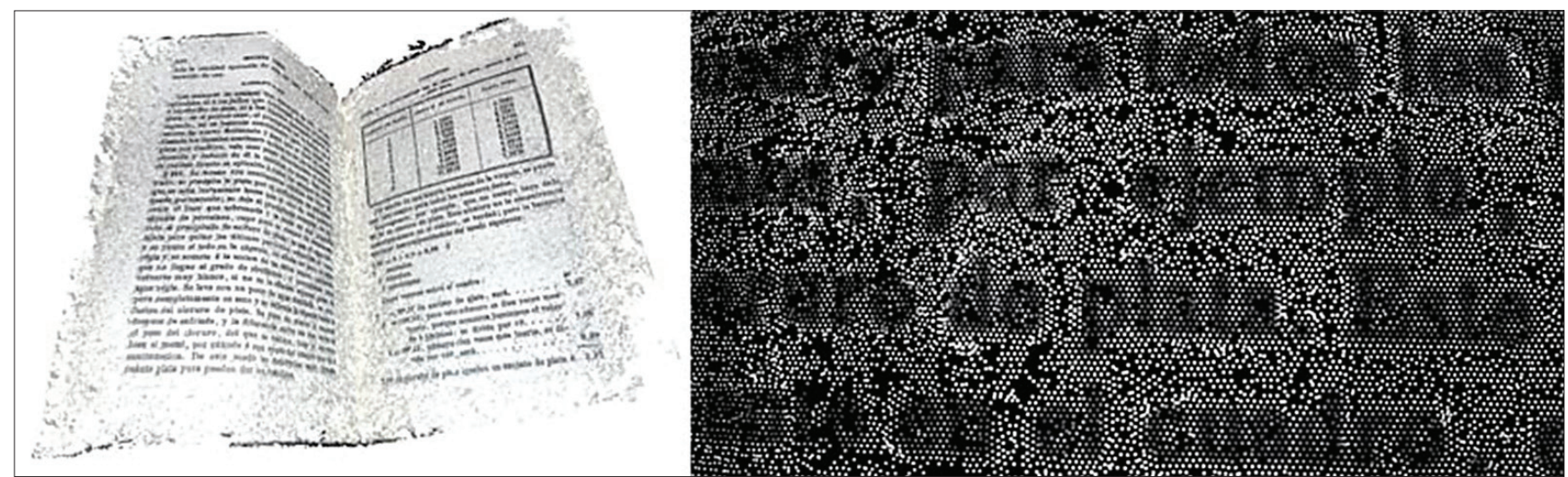

Figura 7. Nube densa 


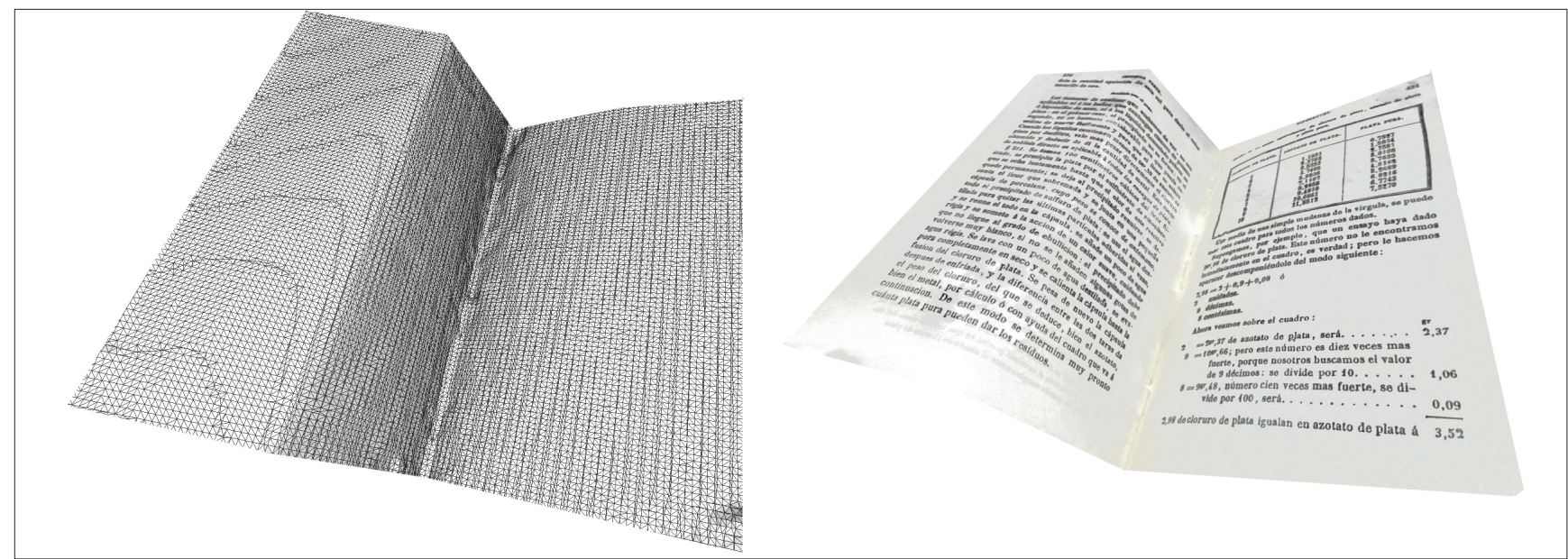

Figura 8. Malla de la superficie del objeto junto a su virtualización texturada

perficies del documento representadas en cada vista.

- Imágenes RTI de las vistas más relevantes del documento en alta resolución. Proporcionan un conocimiento preciso de las texturas a un nivel microscópico.

- Anaglifo en alta resolución. Facilita un análisis sobre la pantalla del ordenador de detalles en volumen a nivel microscópico con el uso de unas gafas con filtros de colores rojo y cian.

b) Componentes para divulgación.

- Modelo 3D virtualizado con texturas fotorrealísticas.

- Par estereoscópico fotográfico del documento completo. Facilita la sensación de espacio tridimensional mediante autoestereoscopia, sin ayuda de ningún visor adicional.

De cara a la implementación de estrategias de preservación digital, es recomendable formatear los modelos 3D bajo estándares abiertos, como los que citamos en el siguiente epígrafe, pues facilitarán la migración a formatos futuros no obsoletos; así como el uso de un esquema de metadatos que permita una descripción suficiente de la tecnología aplicada, del objeto digital y de su contenido. Todavía no se dispone de esquemas de metadatos específicos suficientemente completos para este tipo de modelos en documentos, pero hay que mencionar nuevamente el proyecto Carare, que aporta recomendaciones orientadas a la publicación 3D web y 3D pdf para objetos y monumentos, así como un esquema propio basado en diferentes estándares y buenas prácticas (Papatheodorou et al., 2010).

\section{Comunicación y difusión de modelos 3D}

El uso de formatos abiertos estandarizados es importante también de cara a facilitar el acceso universal a los modelos, pues podrán ser visualizados en cualquier navegador web que reconozca estándares $\mathrm{W} 3 \mathrm{C}$, o con aplicaciones complementarias gratuitas de fácil instalación. En este sentido, recomendamos el uso del formato X3D (.x3d), normalizado por la ISO y sucesor de VRML (virtual reality modeling language). Este formato se basa en $\mathrm{xml}$ y puede ser usado como medio de publicación nativa en la Web en conjunción con X3DOM y html5 (Koutsoudis, 2012).

Otros formatos más habituales de amplio uso en la divulgación de modelos 3D patrimoniales son:

- Stanford triangle format (.ply);

- Wavefront file (.obj), formado habitualmente por un archivo .obj y uno .mtl con la información de la textura;

- Universal 3D file (.u3d), codificado en XML y el único formato soportado para construir los modelos 3D pdf;

- Collada (.dae). Es un estándar abierto de reciente aparición, codificado en $\mathrm{xml}$, soportado nativamente para su previsualización por las últimas versiones de Mac OS $X$.

En la difusión de modelos 3D a través de la Web, el tamaño de los archivos puede ser crítico, debiéndose buscar un

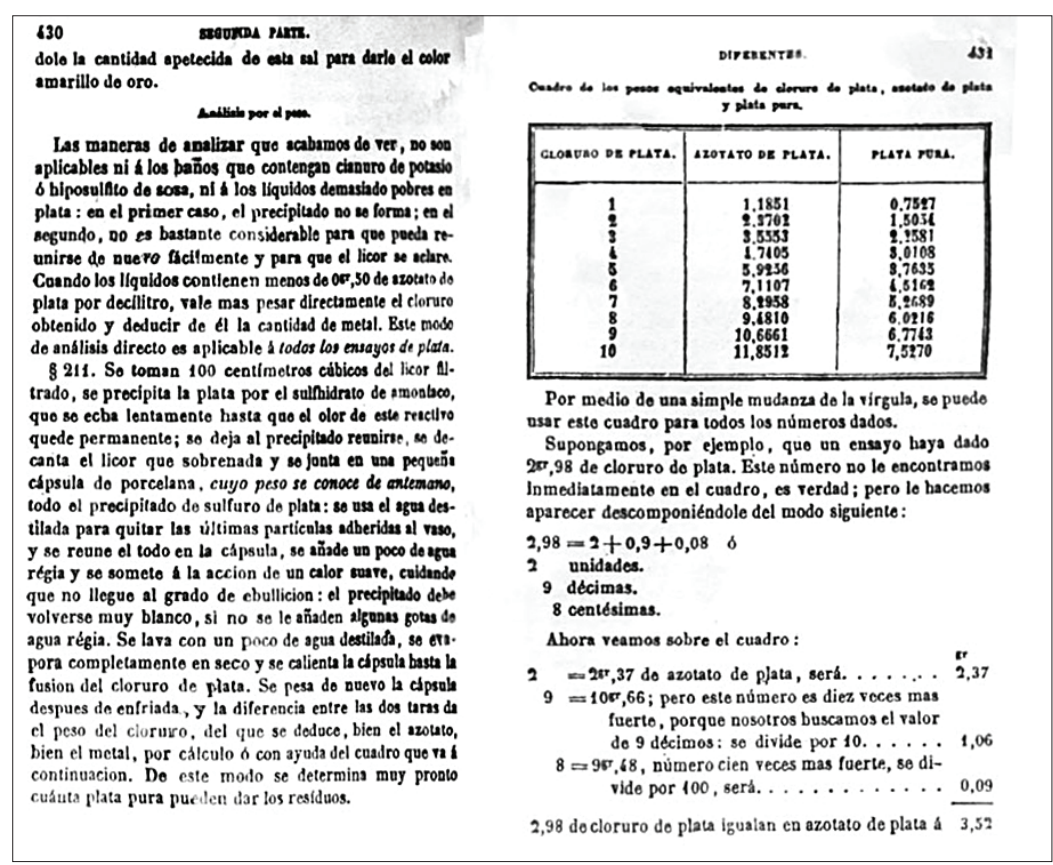

Figura 9. Ortofotografía del objeto modelado 


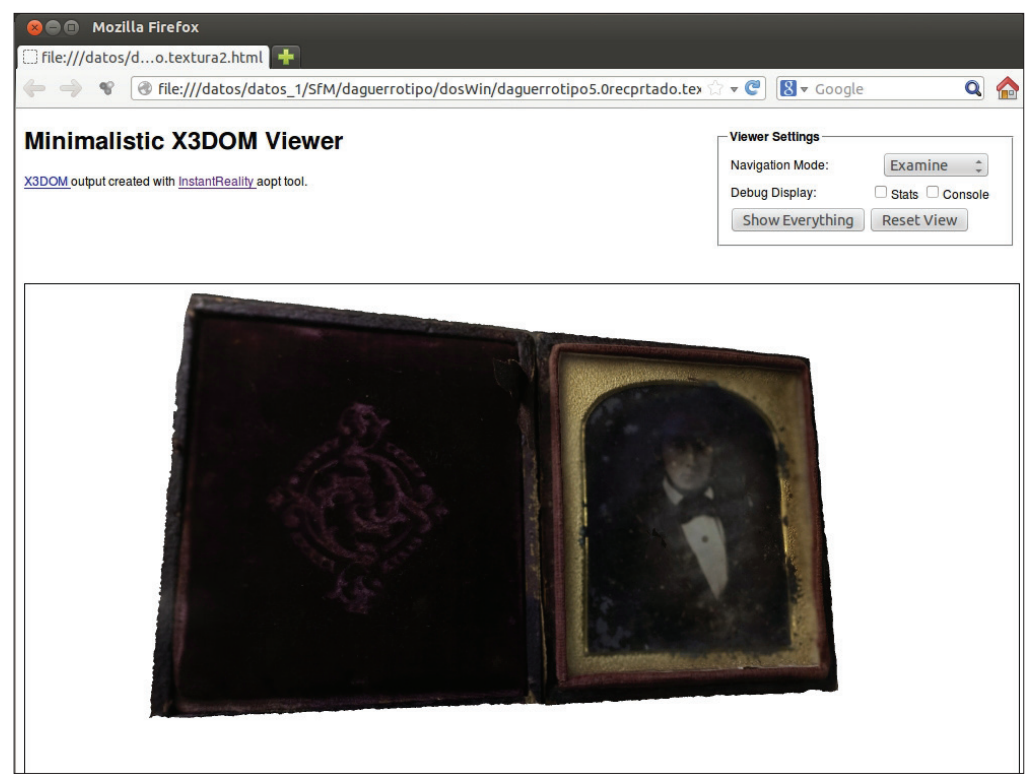

Figura 10. Visualización del modelo de daguerrotipo presentado anteriormente codificado en X3D desde un navegador Firefox, mediante html5 y X3DOM

\section{Notas}

1. ADA, C.145-1. Orig. Perg. $290 \times 415 \mathrm{~mm}$. Proveniente de digitalización de documentos de San Pedro de Arlanza en el Archivo de los Duques de Alba. Consejo Superior de Investigaciones Científicas. Proyecto Arlanza, Once siglos.

2. Obtenido en el Smithsonian Museum Conservation Photography.

http://www.si.edu/mci/EarlyPhotography/ rti_video.html

3. VisualSFM: A visual structure from motion system.

http://homes.cs.washington.edu/ ccwu/ vsfm

4. CloudCompare 3D point cloud and mesh processing software. Open source project. http://www.danielgm.net/cc

\section{Bibliografía}

equilibrio entre resolución y tamaño con el fin de hacerlos fácilmente descargables y visualizables. Los modelos 3D de fácil descarga combinan mallas muy simplificadas con texturas fotorrealísticas, que refuerzan la sensación de detalle y volumen.

Proyectos como Carare ya han comenzado a normalizar la difusión de contenidos 3D (X3D), tanto a nivel de formato como de metadatos descriptivos o técnicos, con el fin de mejorar la compresión de monumentos, objetos o espacios usando modelos 3D o la realidad virtual.

\section{Conclusiones y trabajos futuros}

Una documentación visual completa de un documento patrimonial valioso con volumen o elementos tridimensionales relevantes requiere, junto con imágenes raster bidimensionales, el concurso de tecnologías 3D de diversa naturaleza. A lo largo de este estudio hemos comprobado la utilidad y factibilidad de aplicar tecnologías de fotografía estereoscópica digital, RTI, modelos 3D de nubes de puntos y mallas obtenidos mediante técnicas fotogramétricas o SfM, y ortofotografías derivadas a partir de los anteriores. Esta documentación 3D será una fuente de información valiosa que ayudará al estudioso en la realización de análisis precisos de las características tridimensionales y colorimétricas de los documentos, pero también facilitará su divulgación a través de la Web, debido a su alto impacto visual.

Gracias al auge de las filosofías del código abierto y licencias de dominio público, y la aparición de tecnologías de captura y procesado más sencillas y usables con dispositivos de menor coste, como SfM, se pueden acometer tareas de modelado 3D con altos niveles de precisión y eficiencia.

El formato estándar X3D en combinación con html5 y X3DOM facilita la difusión de los modelos a través de la Web, al poderse visualizar sin el requerimiento de complementos adicionales desde navegadores web de uso común.
Alkhadour, Wissam; Ipson, Stan; Zraqou, Jamal; Qahwaji, Rami; Haigh Jackie (2009). "Creating a color anaglyph from a pseudo-stereo pair of images".

http://www.zuj.edu.jo/conferences/ICIT09/PaperList/ Papers/Image\%20and\%20Signal\%20Processing/578.pdf

Bianco, Gianfranco; Bruno, Fabio; Tonazzini, Anna; Salerno, Emanuele; Savino, Pasquale; Zitová, Barbara; Sroubek, Filip; Console, Elena (2010). "A framework for virtual restoration of ancient documents by combination of multispectral and 3D imaging". En: Eurographics Italian Chapter conf. http://goo.gl/O7wno

Brown, Michael S.; Sun, Mingxuan; Yang, Ruigang; Yun, Lin; Seales, W. Brent (2007). "Restoring 2D content from distorted documents". IEEE Transactions on pattern analysis and machine intelligence, v. 29, n. 11, pp. 1904-1916.

Cultural heritage imaging (2012). "Reflectance transformation imaging (RTI)"

http://culturalheritageimaging.org/Technologies/RTI

Earl, Graeme et al. (2011). "Reflectance transformation imaging systems for ancient documentary artefacts". Electronic visualisation and the arts (EVA 2011), pp. 147-154. http://ewic.bcs.org/upload/pdf/ewic_ev11_s8paper3.pdf

Edirisinghe, Eran; Jiang, Jianmin (2000). "Stereo imaging, an emerging technology". En: Procs of SSGRR 2000: Int conf on advances in infrastructure for e-business, science, and education on the internet.

http://www.loreti.it/Download/PDF/3D/067.pdf

Farnsworth, Robert J. "Amateur anaglyphs". http://courses.washington.edu/photogra/Spring2006/ Anaglyphs.pdf

loannides, Marinos; Fritsch, Dieter; Leissner, Johanna; Davies, Rob; Remondino, Fabio; Caffo, Rossella (eds.) (2012). "Progress in cultural heritage preservation". 4th Intl conf Eu- 
roMed 2012. Limassol, Cyprus, Oct. 29-Nov. 3, 2012. Procs". Springer-Verlag. ISBN: 9783642342332

Kazhdan, Michael; Bolitho, Matthew; Hoppe, Hugues (2006). "Poisson surface reconstruction". En: Polthier, Konrad; Sheffer, Alla (eds.). Eurographics symposium on geometry processing.

http://faculty.cs.tamu.edu/schaefer/teaching/689_ Fall2006/poissonrecon.pdf

Koutsoudis, Anestis ([2012]). "Bringing cultural heritage 3D content to the web using the X3DOM framework" http://carare.eu/ita/Media/Files/Bringing-3D-Content-tothe-Web-Using-X3DOM

Mudge, Mark; Malzbender, Tom; Schroer, Carla; Lum, Marlin (2006). "New reflection transformation imaging methods for rock art and multiple-viewpoint display". En: The $7^{\text {th }}$ IntI symposium on virtual reality, archaeology and cultural heritage VAST.

http://dx.doi.org/10.2312/VAST/VAST06/195-202

Pal, Kazim; Terras, Melissa; Weyrich, Tim (2013). “Interactive exploration and flattening of deformed historical documents". En: Eurographics 2013, v. 32, n. 2.

http://web4.cs.ucl.ac.uk/staff/t.weyrich/projects/gpb/ parchexplore.pdf

Papatheodorou, Christos; Carlisle, Phil; Ertmann-Christiansen, Christian; Fernie, Kate (2010). "The CARARE metadata schema".

http://www.carare.eu/eng/Resources/CARARE-metadataschema-outline-v1.0

Pletinckx, Daniel (2011). "Europeana and 3D". En: Procs Congress of the Int/ Society for Photogrammetry and Remote Sensing. Technical session 9 - Interactive online 3D models. Vol. XXXVIII-5/W16. $2^{\text {nd }}-4^{\text {th }}$ March 2011 Trento, Italy. http://www.int-arch-photogramm-remote-sens-spatial-
inf-sci.net/XXXVIII-5-W16/483/2011/isprsarchives-XXXVIII5-W16-483-2011.html

Piquette, Kathryn E.; Crowther, Charles (2011). “Developing a reflectance transformation imaging (RTI) system for inscription documentation in museum collections and the field. Case studies on ancient Egyptian and classical material". http://www.digitalclassicist.org/wip/wip2011-01kp.pdf

Stanco, Filippo; Battiato, Sebastiano; Gallo, Giovanni (2011). Digital imaging for cultural heritage preservation: analysis, restoration, and reconstruction of ancient artworks. CRC Press Inc. ISBN: 9781439821732

StereoGraphics Corporation (1997). "Stereographics developers' handbook".

http://www.cs.unc.edu/Research/stc/FAQs/Stereo/stereohandbook.pdf

Tian, Yuandong; Narasimhan, Srinivasa (2011). "Rectification and 3D reconstruction of curved document images". En: Proc. IEEE Conf on computer vision and pattern recognition, pp. 377-384.

http://www.cs.cmu.edu/ ILIM/projects/IM/document_ rectification/cvpr2011_YuandongTian.pdf

Wu, Changchang (2011). "A visual structure from motion system". http://www.cs.washington.edu/homes/ccwu/vsfm

Wu, Changchang; Agarwal, Sameer; Curless, Brian; Seitz, Steven (2011). "Multicore bundle adjustment". En: Procs. IEEE Conf on computer vision and pattern recognition, pp. 3057-3064. http://grail.cs.washington.edu/projects/mcba/pba.pdf

Zhang, Li; Tan, Chew-Lim (2005). "Warped image restoration with applications to digital libraries". En: Procs of $8^{\text {th }}$ Intl conf on document analysis and recognition, v. 1, pp. 192196.

http://dx.doi.org/10.1109/ICDAR.2005.252

\section{Ya ha salido el}

\section{Anuario ThinkEPI 2013}

272 páginas de análisis de tendencias en información, documentación y comunicación

\section{Formulario de compra:}

http://www.thinkepi.net/pedido

Información y pedidos:

Isabel Olea

epi.iolea@gmail.com

$+34-608491521$

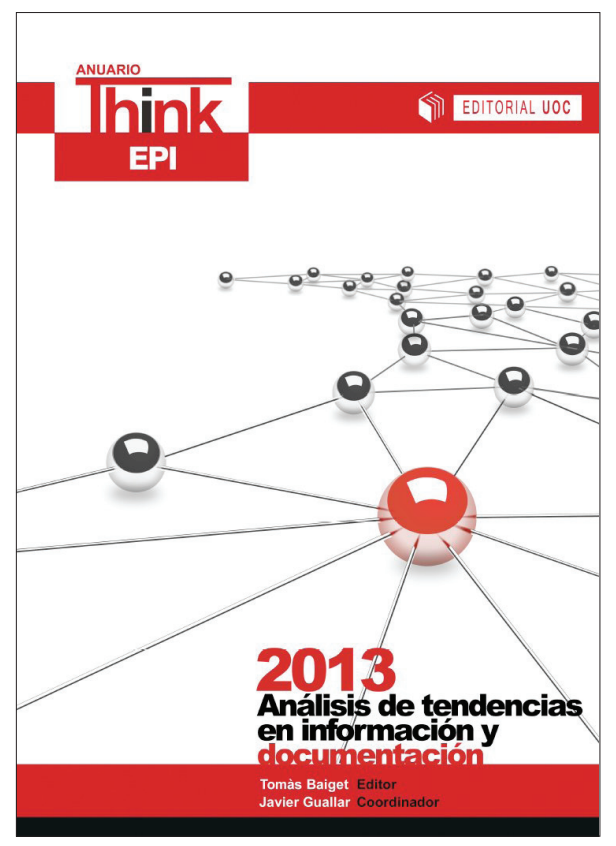

